\title{
Clinical Significance of Galactose-Deficient IgA1 by KM55 in Patients with IgA Nephropathy
}

\author{
Kai Zhang Qiongqiong Li Yaru Zhang Wenya Shang Li Wei \\ Hongfen Li Shan Gao Tiekun Yan Junya Jia Youxia Liu Shan Lin \\ Department of Nephrology, Tianjin Medical University General Hospital, Tianjin, PR China
}

\section{Keywords}

Galactose-deficient IgA1 - KM55 antibody · Complement activation · IgA nephropathy

\begin{abstract}
Background: Aberrant galactose-deficient $\lg A 1$ molecules (Gd-lgA1) are important causal factors in IgA nephropathy (IgAN); however, the detection of Gd-IgA1 in IgAN is complicated and instable. A monoclonal antibody, KM55, which specifically recognizes Gd-IgA1 has been developed. In the present study, we further explored the clinical significance of Gd-IgA1 using KM55. Methods: In this study, we enrolled 75 patients with IgAN and 80 healthy controls and detected the plasma Gd-IgA1 levels using the KM55 ELISA method. We also stained mesangial Gd-IgA1 deposition using KM55. Results: We observed that the levels of plasma Gd-IgA1 in IgAN patients were elevated compared to the corresponding levels of healthy controls. Patients were divided into 2 groups based on the median of Gd-lgA1. Patients with high Gd-IgA1 levels had significantly higher levels of uric acid (UA) and IgA. The other clinical manifestations demonstrated that there were no differences in age, sex, blood pressure, initial proteinuria, hematuria, estimated glomerular filtration rate and Oxford pathological classification between the 2 groups of patients. In addition, positive correlations were observed between $\mathrm{Gd}-\lg A 1$ and Bb, C3a, C4d and MAC. Mesangial Gd-IgA1 was positive in IgAN but negative in the normal renal tissue adjacent to neoplasm. We next analyzed the correlation between plasma Gd-IgA1 and mesangial Gd-IgA1 deposition. The results showed that a high level of plasma Gd-IgA1 was related to the deposition of mesangial Gd-IgA1, although the difference was not significant. Conclusion: We verified the elevated level of plasma and mesangial Gd-IgA1 in patients with IgAN by KM55, which provided an alternative, easy, and reliable tool for diagnosis and activity assessment of IgAN. The level of plasma Gd-IgA1 positively correlated with levels of UA, total IgA levels, and complement activation products.
\end{abstract}

K.Z. and Q.L. contributed equally to this work. 


\section{Kidney \\ Blood Pressure \\ Research}

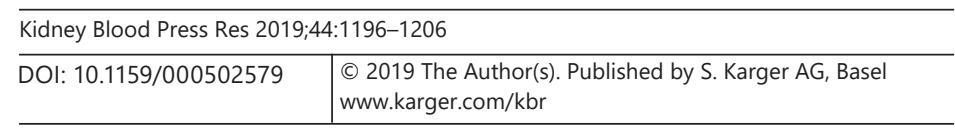

Zhang et al.: Gd-lgA1 by KM55 in IgAN

\section{Introduction}

IgA nephropathy (IgAN) is the most common primary glomerulonephritis that is used worldwide, especially in Asian countries [1,2]. Approximately $20-30 \%$ of the patients with IgAN will progress to end-stage renal disease within 20 years [3, 4]. However, the diagnosis of IgAN requires a renal biopsy. As we all know, renal biopsy has its risks, sometimes deadly complications, non-invasive diagnostic methods alternative to the common biopsy are needed. Recently, increasing evidence has indicated that galactose-deficient IgA1 (Gd-IgA1) molecules are the most convincing trigger factors for pathogenesis of IgAN [5-7]. Furthermore, the researchers have reported that high levels of Gd-IgA1 were associated with disease progression [8, 9]. However, evidence of Gd-IgA1 as a biomarker remains controversial [10]. The main reason for the controversy is the absence of a definitive assay. At present, the detection of the Gd-IgA1 hinge region in IgAN required either mass spectroscopy [11] or lectin-based assays [12]. The limitations of these methods are that its bioactivity and stability depend strongly on the purified lectin. In order to detect and quantify circulating levels of Gd-IgA1 robustly and accurately, KM55, a monoclonal antibody, which specifically recognizes Gd-IgA1, has been developed. In their studies, Suzuki and colleagues [13, 14] had demonstrated that this antibody specifically recognized Gd-IgA1 in serum and glomeruli just like using HAA lectin-based assay. However, the commercially available KM55 antibody is not widely validated.

In this study, we detected plasma and mesangial Gd-IgA1 using KM55 and clarified the clinical significance of Gd-IgA1 in Chinese patients with IgAN.

\section{Patients and Methods}

\section{Subjects}

Seventy-five IgAN patients diagnosed in Tianjin Medical University General Hospital from March 2017 to January 2018 were enrolled in this study. The diagnosis was based on the deposition of IgA in the glomerular mesangium by immunofluorescence detection, as well as the lack of clinical or serological evidence of other inflammatory conditions, such as Henoch Schoenlein purpura, systemic lupus erythematosus. At the same time, 80 healthy volunteers whose age and gender matched with patients were recruited. Plasma was collected from all individuals in this study, for patients at the time of renal biopsy. The plasma samples were stored in aliquots at $-80^{\circ} \mathrm{C}$ for the subsequent use. Clinical information, including age, gender, 24-h urine protein excretion, blood pressure and total IgA levels, were collected at the time of renal biopsy. The estimated glomerular filtration rate (eGFR) was calculated using the chronic kidney disease epidemiology collaboration creatinine equation [15]. The histological lesions were classified according to the Oxford classification system. The Medical Ethics Committee of Tianjin medical university general hospital approved the study protocol and informed written consent was obtained from all individuals.

\section{Assay of Plasma Gd-IgA1}

Plasma Gd-IgA1 levels were detected using the ELISA kit according to the manufacturer's specifications (IBL, Japan). Plasma samples were diluted in proportions of 1:50 with EIA buffer and incubation for 60 min at R.T. with plate lid. Then after washing 4 times with the wash buffer, the prepared labelled antibodies were incubated for $30 \mathrm{~min}$. Plates were washed 3 times and added to $50 \mu \mathrm{L}$ TMA solution incubation for $30 \mathrm{~min}$ in dark. At last, the colour reaction was stopped and the absorbance was measured at 450/625 nm with an EL312 BioKinetics microplate reader (Bio-Tek Instruments, USA). 


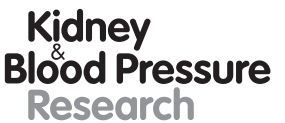

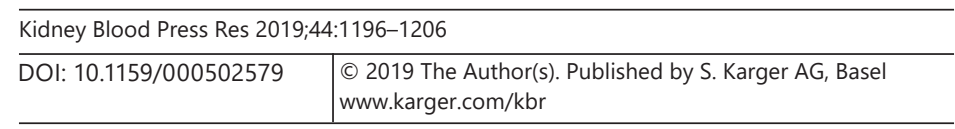

Zhang et al.: Gd-IgA1 by KM55 in IgAN

\section{Plasma Complement Component Levels}

The concentrations of human complement components, including C $3 \mathrm{a}, \mathrm{C} 4 \mathrm{~d}, \mathrm{Bb}$ and $\mathrm{C} 5 \mathrm{~b}-9$ (MAC), were determined according to the manufacturer's instructions by ELISA (Quidel, USA). The absorbance was measured at $405 \mathrm{~nm}$ with an EL312 Bio-Kinetics microplate reader (Bio-Tek Instruments, USA).

\section{Immunohistochemistry Staining of Mesangial Gd-IgA1}

Renal specimens were evaluated with direct immunohistochemistry and were forwarded to 2 pathologists. Both pathologists examined the biopsies independently, being blinded to each other as well as to the patients' clinical data. The renal tissue sections of IgAN patients and adjacent non-neoplastic tissues were collected;5-mm-thick formalin-fixed paraffinembedded renal biopsies were deparaffinized and hydrated. Antigen retrieval with subtilisin A (Sigma, Japan) was performed at room temperature for $2 \mathrm{~h}$. The rat monoclonal anti-human Gd-IgA1 antibody (dilution 1:100, Immuno-Biological Laboratories, Japan) was added to each coverslip directly. Antibodies were incubated overnight at $4{ }^{\circ} \mathrm{C}$. After sufficient wash with phosphate-buffered saline, horseradish peroxidase - conjugated polyclonal goat anti-rat IgG (Life Technologies, USA) was added at $37^{\circ} \mathrm{C}$ for 30 min. Finally, sections were examined using light microscopy. The biopsies with at least 8 glomeruli were used for the quantification of intensity of mesangial Gd-IgA1. The intensity of Gd-IgA1 in mesangial areas was assessed as 1, weak; 2 , mild; 3, moderate; 4 . Severe (as shown in online suppl. Fig. 1; for all online suppl. material, see www.karger.com/doi/10.1159/000502579) [16].

\section{Statistical Analyses}

Normal distribution quantitative variables were expressed as means and SDs and compared by an independent-samples $t$ test. For non-normally distributed variables, we used median and interquartile range and analyzed with the Mann-Whitney U test. Categorical data was summarized by percentages. A two-sided $p$ value $<0.05$ was considered statistically significant. All statistical tests were performed using SPSS version 16.0.

\section{Results}

\section{Baseline Clinical, Laboratory and Pathological Data}

Among the 75 IgAN patients, there were 40 (53.3\%) males with mean age of $39 \pm 14$ at the time of kidney biopsy. The mean systolic blood pressure was $125 \pm 18 \mathrm{~mm} \mathrm{Hg}$. Biopsy revealed that the mean proteinuria level and total IgA were $1.80 \mathrm{~g} /$ day and $330.97 \mathrm{mg} / \mathrm{dL}$ respectively. The average eGFR and uric acid (UA) were $92.84 \pm 33.57 \mathrm{~mL} / \mathrm{min}$ per $1.73 \mathrm{~m}^{2}$ and $351.7 \pm 113 \mu \mathrm{mol} / \mathrm{L}$ respectively. The grading of the pathological lesions by Oxford classification is shown in Table 1.

\section{Plasma Gd-IgA1 Levels}

Using the GalNAc-specific monoclonal antibody KM55, we examined the plasma levels of Gd-IgA1 in patients with IgAN and in those in the healthy control. In our cohorts, patients with IgAN had significantly higher average Gd-IgA1 levels $(11.09 \pm 4.87 \mu \mathrm{g} / \mathrm{mL})$ compared with those in the healthy control $(7.20 \pm 4.00 \mu \mathrm{g} / \mathrm{mL}, p<0.001$; Fig. 1$)$.

\section{Association of Gd-IgA1 with Clinical Characteristics of IgAN}

After the identification of increased Gd-IgA1 levels in patients with IgAN, we further explored its association with clinical features in patients with IgAN. We classified the patients into 2 groups according to the median value of Gd-IgA1 levels (median value: $10.32 \mu \mathrm{g} / \mathrm{mL}$ ). 
Table 1. Baseline characteristics of 75 patients with IgAN

\begin{tabular}{lc}
\hline & Mean \pm SD (range or percent) \\
\hline Male, \% & $40(53.3)$ \\
Age, years & $39 \pm 14$ \\
SBP, mm Hg & $125 \pm 18$ \\
Follow-time, months & $5.5(0-24)$ \\
eGFR, mL/min/1.73 mm m $^{2}$ & $92.84 \pm 33.57$ \\
Scr, $\mu$ mol/L & $93.25 \pm 50.00$ \\
UA, $\mu$ mol/L & $351.7 \pm 113$ \\
ALB, g/L & $37.57 \pm 5.14$ \\
Urinary protein, g/24 h & $1.80 \pm 1.71$ \\
Urinary RBC, /HP & $37.89 \pm 51.65$ \\
Serum IgA, mg/dL & $330.97 \pm 121.90$ \\
Serum C3, mg/dL & $90.04 \pm 18.14$ \\
Oxford classification, $n$ (\%) & \\
$\quad$ M0/M1 & $75(100) / 0(0)$ \\
$\quad$ E0/E1 & $49(65.3) / 26(34.7)$ \\
S0/S1 & $33(44) / 42(56)$ \\
T0/T1/T2 & $29(38.7) / 40(53.3) / 6(8)$ \\
C0/C1/C2 & $22(29.3) / 42(56.0) / 11(14.7)$
\end{tabular}

IgAN, IgA nephropathy; SBP, systolic blood pressure; eGFR, estimated glomerular filtration rate; Scr, serum creatinine; UA, uric acid; ALB, serum albumin; RBC, red blood cells; M, mesangial cell proliferation; E, endothelial cell proliferation; S, glomerular segmental sclerosis; T, tubular atrophy, interstitial fibrosis; C, crescentic lesion.

Table 2. Baseline characteristics of high level serum Gd-IgA1 group and low level serum Gd-IgA1 group in patients with IgAN

\begin{tabular}{|c|c|c|c|}
\hline Characteristics & $\begin{array}{l}\text { High Gd-IgA1 group } \\
(\text { Gd-IgA1 }>10.32 \mu \mathrm{g} / \mathrm{mL})\end{array}$ & $\begin{array}{l}\text { Low Gd-IgA1 group } \\
\text { (Gd-IgA1 } \leq 10.32 \mu \mathrm{g} / \mathrm{mL} \text { ) }\end{array}$ & $p$ value \\
\hline Age, years & $40.08 \pm 12.67$ & $38.27 \pm 14.67$ & 0.30 \\
\hline Gender, male, \% & $23(62)$ & $16(43)$ & 0.10 \\
\hline eGFR, $\mathrm{mL} / \mathrm{min} / 1.73 \mathrm{~mm}^{2}$ & $86.83 \pm 30.80$ & $99.76 \pm 35.35$ & 0.09 \\
\hline $\mathrm{Scr}, \mu \mathrm{mol} / \mathrm{L}$ & $97.82 \pm 45.0$ & $87.43 \pm 54.31$ & 0.36 \\
\hline $\mathrm{UA}, \mu \mathrm{mol} / \mathrm{L}$ & $391.11 \pm 120.50$ & $325.62 \pm 84.59$ & 0.009 \\
\hline ALB, $g / L$ & $37.73 \pm 4.80$ & $37.81 \pm 5.40$ & 0.95 \\
\hline Urinary protein, $\mathrm{g} / 24 \mathrm{~h}$ & $1.81 \pm 1.69$ & $1.81 \pm 1.75$ & 0.99 \\
\hline Urinary RBC, /HP & $29.90 \pm 34.53$ & $45.89 \pm 64.19$ & 0.19 \\
\hline Serum IgA, mg/dL & $397.00 \pm 119.00$ & $271.09 \pm 91.25$ & $<0.0001$ \\
\hline Serum C3, mg/dL & $90.44 \pm 18.08$ & $90.04 \pm 18.60$ & 0.93 \\
\hline \multicolumn{4}{|l|}{ Oxford classification, $n(\%)$} \\
\hline M0/M1 & $0(0) / 37(100)$ & $0(0) / 38(100)$ & 1.0 \\
\hline E0/E1 & $25(67.6) / 12(32.4)$ & $24(63.2) / 14(36.8)$ & 0.69 \\
\hline S0/S1 & $17(45.9) / 20(54.1)$ & $16(42.1) / 22(57.9)$ & 0.74 \\
\hline $\mathrm{T} 0 / \mathrm{T} 1 / \mathrm{T} 2$ & $16(43.2) / 19(51.4) / 2(5.4)$ & $13(34.2) / 21(55.3) / 4(10.5)$ & 0.59 \\
\hline $\mathrm{C} 0 / \mathrm{C} 1 / \mathrm{C} 2$ & $11(29.7) / 23(62.2) / 11(14.9)$ & $11(28.9) / 19(50) / 8(21.1)$ & 0.27 \\
\hline
\end{tabular}

IgAN, IgA nephropathy; Gd-IgA1, galactose-deficient IgA1; eGFR, estimated glomerular filtration rate; Scr, serum creatinine; UA, uric acid; ALB, serum albumin; RBC, red blood cells; M, mesangial cell proliferation; E, endothelial cell proliferation; S, glomerular segmental sclerosis; T, tubular atrophy, interstitial fibrosis; C, crescentic lesion. 
Fig. 1. Comparison of patients with IgAN and healthy control on the plasma level of Gd-IgA1. GdIgA1, galactose-deficient IgA1; IgAN, IgA nephropathy.

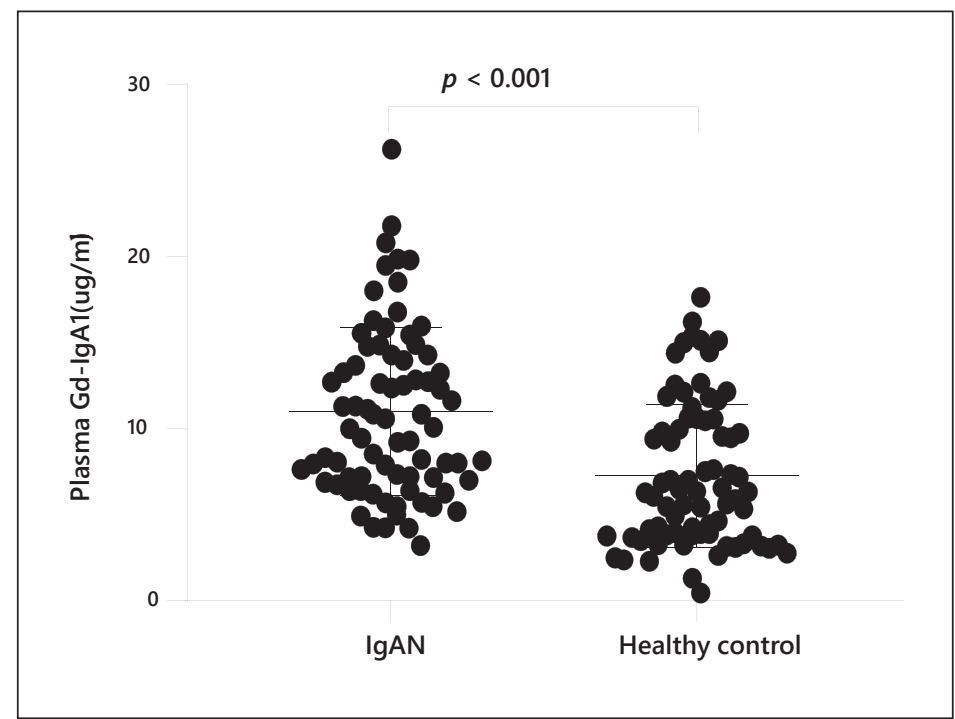

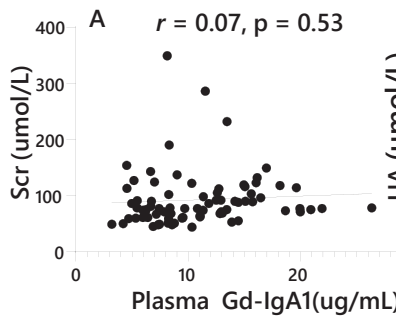

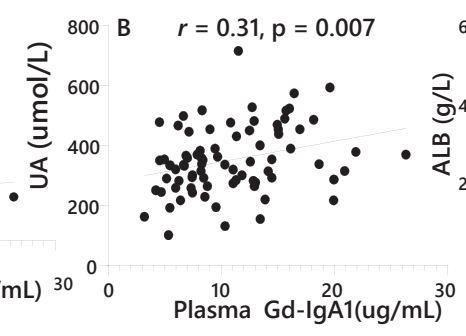

60 C $r=-0.09, \mathrm{p}=0.44$
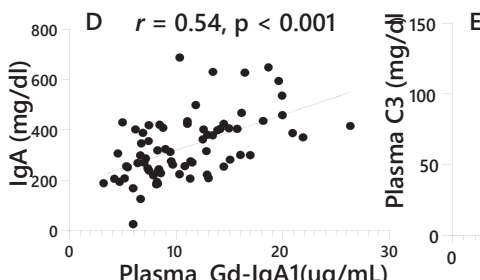

$r=0.006, \mathrm{p}=0.95$
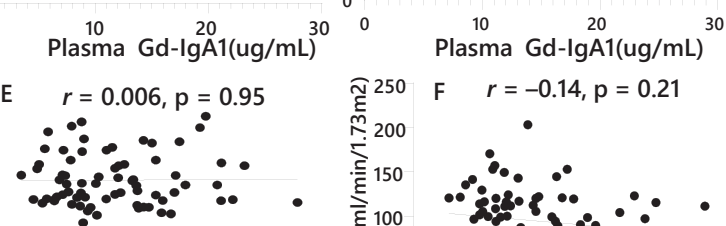

Plasma Gd-lgA1(ug/mL)
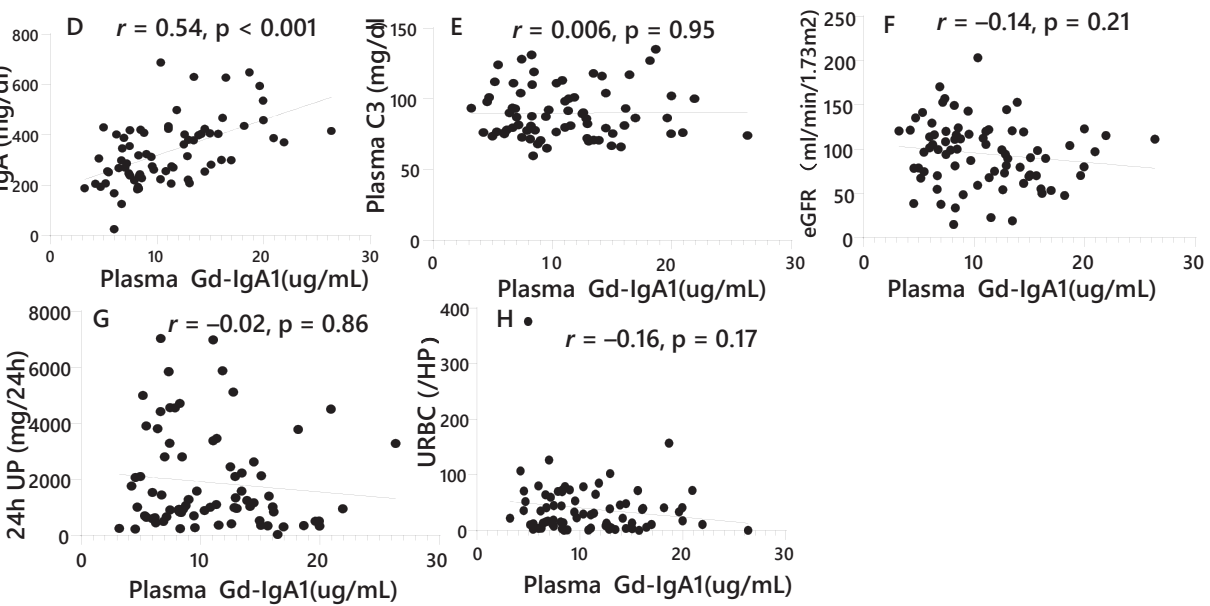

400

Plasma Gd-lgA1(ug/mL)

$\mathrm{H} \bullet r=-0.16, \mathrm{p}=0.17$

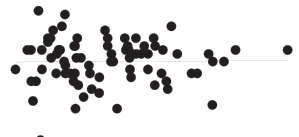

足 300
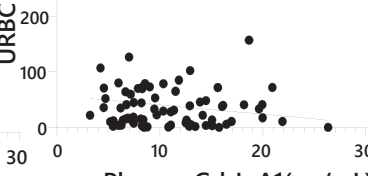

Plasma Gd-lgA1(ug/mL)

Fig. 2. Correlation analysis of Gd-IgA1 with clinical characteristics of IgAN patients. A Correlation analysis of serum creatinine and plasma Gd-IgA1 levels. B Correlation analysis of UA and plasma Gd-IgA1 levels. C Correlation analysis of ALB and plasma Gd-IgA1 levels. D Correlation analysis of plasma IgA and plasma GdIgA1 levels. E Correlation analysis of plasma C3 and plasma Gd-IgA1 levels. F Correlation analysis of eGFR and plasma Gd-IgA1 levels. G Correlation analysis of 24 h proteinuria and plasma Gd-IgA1 levels. H Correlation analysis of Urine blood cells and plasma Gd-IgA1 levels. Scr, serum creatinine; Gd-IgA1, galactose-deficient IgA1; UA, uric acid; ALB, serum albumin; eGFR, estimated glomerular filtration rate; URBC, urinary red blood cells. 


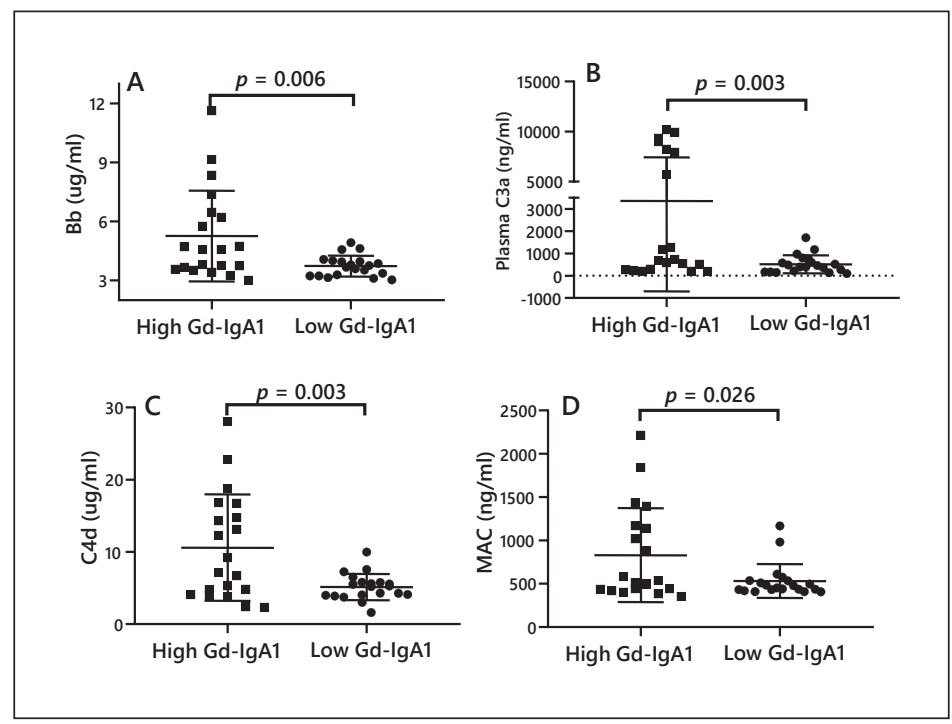

Fig. 3. Comparison of complement fragment levels among the IgA patients with High Gd-IgA1 levels and Low Gd-IgA levels. Among the 40 patients with IgAN, patients with high Gd-IgA1 levels also had higher levels of Bb (High Gd-IgA1 vs. Low Gd-IgA1: 4.56, 3.60-6.40 $\mu \mathrm{g} / \mathrm{mL}$ vs. 3.71, 3.24-3.99 $\mu \mathrm{g} / \mathrm{mL}, p=0.006$, A), C3a (High Gd-IgA1 vs. Low Gd-IgA1: 710, 268-8,177 ng/mL vs. 422, 182-713 ng/mL, $p=0.003$, B), C4d (High Gd-IgA1 vs. Low Gd-IgA1: 8.16, 4.23-16.24 $\mu \mathrm{g} / \mathrm{mL}$ vs. 5.21, 3.99-5.78 $\mu \mathrm{g} / \mathrm{mL}, p=0.003, \mathbf{C}$ ), and MAC (High Gd-IgA1 vs. Low Gd-IgA1: 524, 438-1,161 ng/mL vs. 464, 432-536 ng/mL, $p=0.02$, D). Gd-IgA1, galactose-deficient IgA1; MAC, membrane attack complex.

We found that patients with high Gd-IgA1 levels had significantly higher levels of UA ( $p=$ 0.009 , Table 2$)$ and IgA ( $p<0.001$, Table 2$)$. The other clinical manifestations demonstrated that there were no differences in age, sex, blood pressure, initial proteinuria, hematuria, eGFR and Oxford pathological classification between the 2 groups of patients. In addition, we found that high Gd-IgA1 levels were correlated with high UA levels ( $r=0.31, p=0.007$; Fig. 2B) and total IgA levels ( $r=0.54, p<0.001$; Fig. $2 \mathrm{~d}$ ), while not correlated with other clinical manifestations (Fig. 2A, C, E-H). Furthermore, no correlation was found between eGFR and plasma UA in our study ( $r=-0.13, p=0.25$, online suppl. Fig. 2$)$.

\section{Association of Gd-IgA1 with Complement Fragment Levels}

We randomly selected 40 patients with IgAN to examine the correlation between plasma Gd-IgA1 and complement activation products. In the group of patients with high Gd-IgA1 levels, Bb (High Gd-IgA1 vs. Low Gd-IgA1: 4.56, 3.60-6.40 $\mu \mathrm{g} / \mathrm{mL}$ vs. 3.71, 3.24-3.99 $\mu \mathrm{g} / \mathrm{mL}$, $p=0.006$; Fig. 3A), C3a (High Gd-IgA1 vs. Low Gd-IgA1: 710, 268-8,177 ng/mL vs. 422, 182-713 ng/mL, $p=0.003$; Fig. 3B), C4d (High Gd-IgA1 vs. Low Gd-IgA1: 8.16, 4.23-16.24 $\mu \mathrm{g} / \mathrm{mL}$ vs. $5.21,3.99-5.78 \mu \mathrm{g} / \mathrm{mL}, p=0.003$; Fig. 3C), and membrane attack complex (MAC, High Gd-IgA1 vs. Low Gd-IgA1: 524, 438-1,161 ng/mL vs. 464, 432-536 ng/mL, $p=0.02$; Fig. 3D) levels were significantly higher than patients with low Gd-IgA1 levels. More importantly, we found that high Gd-IgA1 levels were correlated with high $\mathrm{Bb}(r=0.49, p=0.001$; Fig. 4A), C3a $(r=0.56$, $p<0.001$; Fig. 4B), C4d ( $r=0.56, p<0.001$; Fig. 4C), and MAC ( $r=0.40, p=0.01$; Fig. 4D).

\section{Mesangial Gd-IgA1 Deposition}

Furthermore, we randomly selected 15 patients with IgAN to evaluate mesangial Gd-IgA1 deposition. Mesangial Gd-IgA1 was positive in IgAN while negative in the normal renal tissue adjacent to the neoplasm (Fig. 5). 


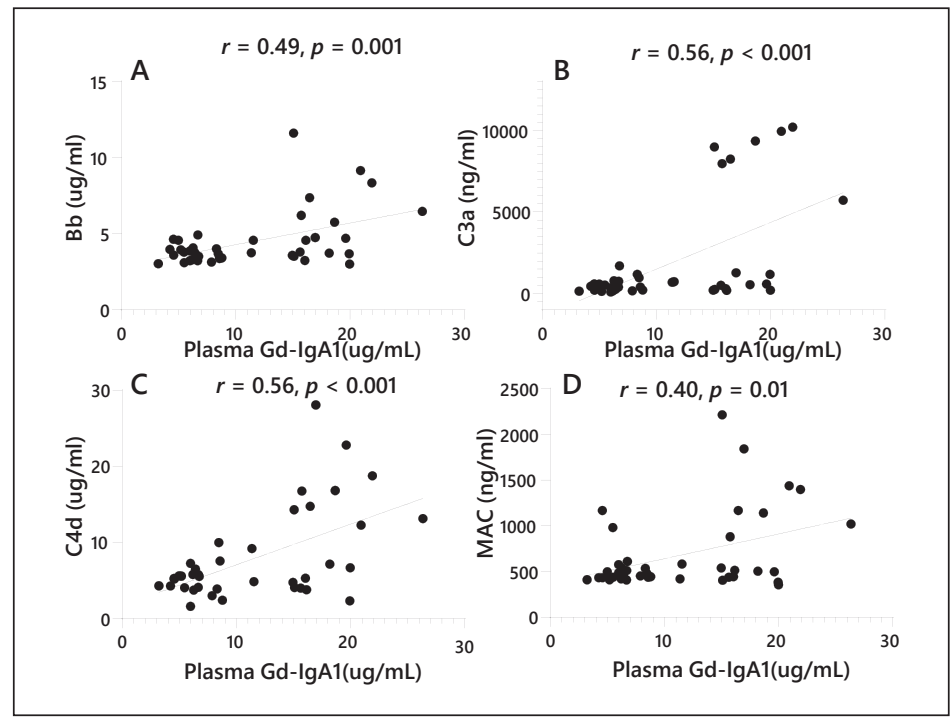

Fig. 4. Correlation analysis of plasma Gd-IgA1 and complement activation products in IgAN patients. Among the IgAN patients with high Gd-IgA1 levels, the levels of Gd-IgA1 were correlated with high Bb $(r=0.49, p=$ $0.001, \mathbf{A})$, C3a $(r=0.56, p<0.001, \mathbf{B}), \mathrm{C} 4 \mathrm{~d}(r=0.56, p<0.001, \mathbf{C})$, and MAC $(r=0.40, p=0.01, \mathrm{D}) . \mathrm{Gd}-\operatorname{IgA} 1$, galactose-deficient IgA1; MAC, membrane attack complex.

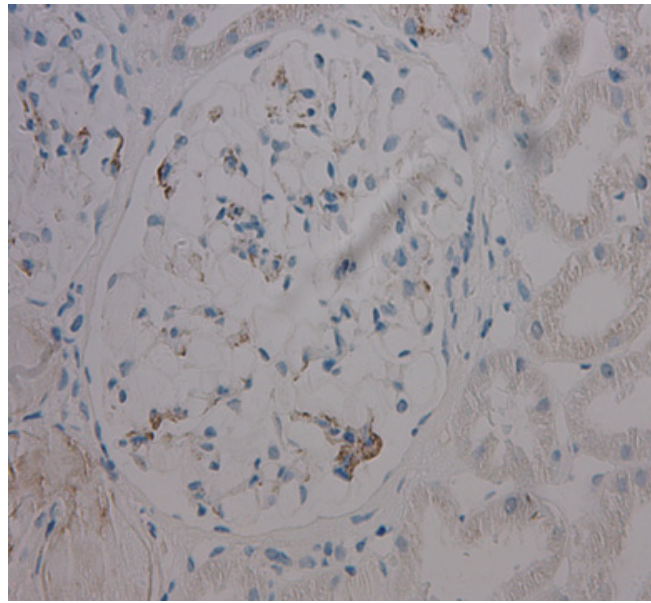

$\operatorname{IgAN}$

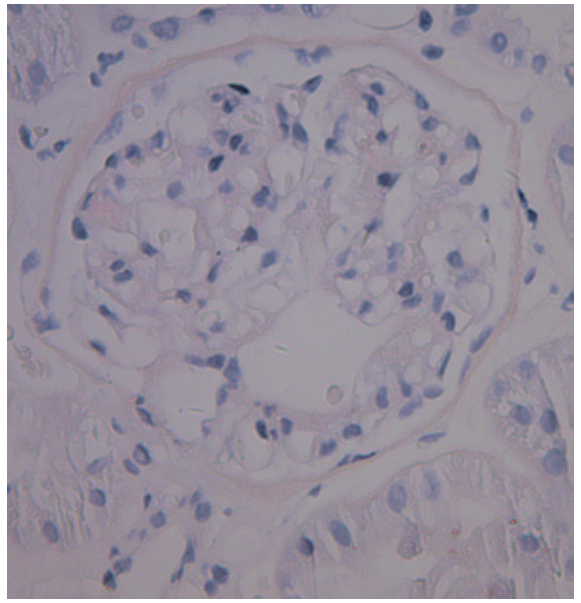

Control

Fig. 5. Immunofluorescent staining for glomerular deposition of Gd-IgA1 in renal biopsy specimens from IgAN patient and healthy control. IgAN, IgA nephropathy.

The Association between Plasma Gd-IgA1 and Mesangial Gd-IgA1 Deposition

We next analyzed the correlation between plasma Gd-IgA1 and mesangial Gd-IgA1 deposition. The results showed that a high level of plasma Gd-IgA1 was related to the deposition of mesangial Gd-IgA1, although the difference was not significant $(r=0.44, p=0.09$, Fig. 6). 
Fig. 6. Correlation analysis of plasma Gd-IgA1 and mesangial Gd-IgA1 deposition. Gd-IgA1, galactose-deficient IgA1.

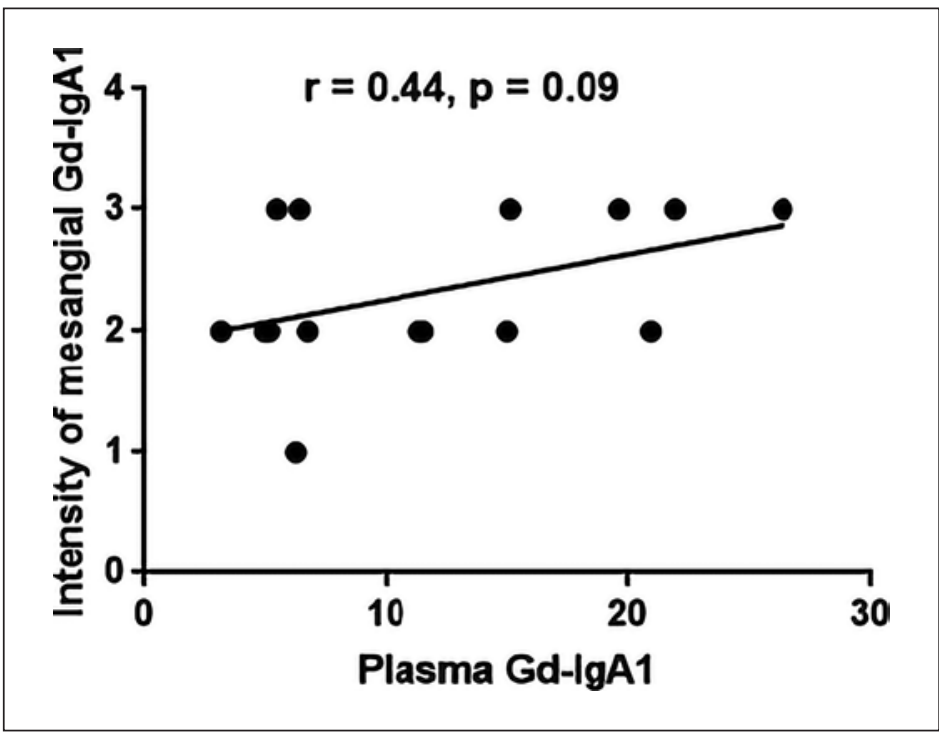

\section{Discussion}

In this study, we measured the levels of Gd-IgA1 in IgAN patients using KM55, which specifically recognized pathogenic Gd-IgA1. Our study at first verified that the novel lectin-independent assay was an easy tool to detect plasma and mesangial Gd-IgA1 in Chinese patients with IgAN. Further, the results showed that in patients with IgAN, the elevated Gd-IgA1 levels were associated with high UA, IgA levels and complement activation products, providing a specific method to better understand the pathogenesis of IgAN.

Since 2001, indirect evidence has accumulated that circulating Gd-IgA1 are the trigger factors for mesangial deposition and subsequent kidney injury in IgAN. Unfortunately, Gd-IgA1 hinge region in IgAN was not easy to detect. $\mathrm{H}$ aspersa lectin, which can specifically recognize the hinge region, is not available in a synthetic form and has to be purified from the snails. The purification process variation of the lectin has hampered the reliability and popularization of assays. Only a handful of well-equipped labs in the world could detect Gd-IgA1 by lectin-based assay $[17,18]$, while others could not. In order to overcome the above-mentioned difficulty and improve stability, Suzuki and colleagues $[13,14]$ obtained a novel and unique monoclonal antibody, KM55, against Gd-IgA1. This method can recognize Gd-IgA1 in patients with IgAN, just like a lectin-based assay. Consistent with Suzuki's report, we also observed elevated plasma Gd-IgA1 levels in our patients with IgAN by this ELISA kit. Moreover, mesangial Gd-IgA1 deposition was specific to IgAN, which was compatible with previous results $[14,19]$. Some healthy controls with relatively high levels of plasma Gd-IgA1 suggested that circulating Gd-IgA1 is not the only factor linked to the onset of IgAN. It indicated that excess Gd-IgA1 in serum and glomerular mesangial area deposits could induce chronic glomerular injuries in IgAN. We found that a high level of plasma Gd-IgA1 was related to the strong deposition of mesangial Gd-IgA1 deposition, although the difference was not significant. Wada et al. [19] found serum Gd-IgA1 positively correlated with mesangial Gd-IgA1 intensity scores in the 50 patients with $\operatorname{IgAN}(r=$ $0.21, p=0.006$ ). As said by Floege, "KM55 would still have specificity similar to that of PLA2R antibodies in membranous nephropathy, which clearly revolutionized that field" [20]. More and more evidence seems to indicate that increased staining for PLA2R in glomeruli of renal biopsies tightly correlates with the presence of PLA2R autoantibodies in the serum, which correlated to 
disease activity, treatment response and prognosis. Whether KM55 played a similar important role in IgAN was required to verify in more patients. Further studies are needed to determine the normal range of Gd-IgA1 in order to distinguish patients with IgAN from healthy persons, similar to PLA2R antibodies.

In a previous study, Gal-deficient IgA1 was closely associated with pathologic phenotypes and prognosis in patients of IgAN $[8,9,21]$. Unfortunately, in our study, no significant difference as for M, E, S, T and C classification was observed between patients with the low and high levels of Gd-IgA1. We found that patients with higher levels of Gd-IgA1 presented with higher levels of UA and total IgA levels.

Accumulating evidence revealed that complement activation has a role in the pathogenesis and progression of IgAN [22-24]. In our study, although the majority of patients had normal levels of complement C3, the plasma levels of complement activation products were much higher in patients with higher levels of Gd-IgA1 than those with low levels of Gd-IgA1. We found that high Gd-IgA1 levels were correlated with an alternative pathway, which contained a high alternative pathway fragment $\mathrm{Bb}$. In recent years, many studies showed that glomeruli from patients with IgAN had significantly higher abundance of components of the classical pathway. We detected the classical pathway fragment $\mathrm{C} 4 \mathrm{~d}$, and found that high Gd-IgA1 levels were correlated with high C4d. Also, high Gd-IgA1 levels were showed to correlate with all 3 pathways product $\mathrm{C} 3 \mathrm{a}$ and terminal pathway complement complex MAC $[25,26]$. Therefore, it is difficult to clarify which complement pathway is critically activated by Gd-IgA1. More studies should be needed to elucidate this question and develop complementtargeting therapy of the disease.

However, the small sample size was the major limitation of the study. Second, our present study was a cross-sectional study. We detected the Gd-IgA1 levels only on the day of renal biopsy for patients with IgAN. Therefore, we cannot currently evaluate the variability of Gd-IgA1 levels over time. Additional evaluation studies in large and long-term follow-up cohorts are required in the future.

\section{Conclusion}

In conclusion, we verified that plasma and mesangial Gd-IgA1 were elevated in patients with IgAN by KM55, which provided an alternative easy and reliable tool for diagnosis and activity assessment of IgAN. The level of plasma Gd-IgA1 positively correlated with levels of $\mathrm{UA}$, total IgA levels and complement activation products.

\section{Acknowledgement}

The authors thank all the study subjects for their participation.

\section{Statement of Ethics}

All subjects provided written informed consents. The study protocol was approved by the Institutional Ethical Committee of Tianjin medical university general hospital. 
Kidney
Blood Pressure
Research

\begin{tabular}{l|l}
\hline Kidney Blood Press Res 2019;44:1196-1206 \\
\hline DOI: 10.1159/000502579 & $\begin{array}{l}\text { ○ 2019 The Author(s). Published by S. Karger AG, Basel } \\
\text { www.karger.com/kbr }\end{array}$ \\
\hline
\end{tabular}

Zhang et al.: Gd-lgA1 by KM55 in IgAN

\section{Disclosure Statement}

The authors have no conflicts of interest to declare.

\section{Funding Sources}

This study is supported by the National Natural Science Foundation (81600553).

\section{Author Contributions}

Y.L. and S.L. conceived the study, and participated in its design and coordination. K.Z., Q.L., Y.Z., W.S., L.W., H.L. and S.G. collected clinical data. K.Z. performed the Gd-IgA1 measurement. T.Y. interpreted the data. Q.L. contributed to the writing of the manuscript. All authors read and approved the final manuscript for submission.

\section{References}

1 Tang SC. An Overview of IgA Nephropathy: 50 Years On. Semin Nephrol. 2018 Sep;38(5):433-4.

2 Moriyama T. Clinical and histological features and therapeutic strategies for IgA nephropathy. Clin Exp Nephrol. 2019 Sep;23(9):1089-99.

3 Lin Y, Jia J, Guo Y, He D, Zhang Y, Wang F, et al. Corticosteroid for IgA Nephropathy: Are They Really Therapeutic? Am J Nephrol. 2018;47(6):385-94.

4 Li X, Liu Y, Lv J, Shi S, Liu L, Chen Y, et al. Progression of IgA nephropathy under current therapy regimen in a Chinese population. Clin J Am Soc Nephrol. 2014 Mar;9(3):484-9.

5 Moldoveanu Z, Wyatt RJ, Lee JY, Tomana M, Julian BA, Mestecky J, et al. Patients with IgA nephropathy have increased serum galactose-deficient IgA1 levels. Kidney Int. 2007 Jun;71(11):1148-54.

6 Novak J, Barratt J, Julian BA, Renfrow MB. Aberrant Glycosylation of the IgA1 Molecule in IgA Nephropathy. Semin Nephrol. 2018 Sep;38(5):461-76.

7 Bagchi S, Lingaiah R, Mani K, Barwad A, Singh G, Balooni V, et al. Significance of serum galactose deficient IgA1 as a potential biomarker for IgA nephropathy: A case control study. PLoS One. 2019 Mar;14(3):e0214256.

8 Zhao N, Hou P, Lv J, Moldoveanu Z, Li Y, Kiryluk K, et al. The level of galactose-deficient IgA1 in the sera of patients with IgA nephropathy is associated with disease progression. Kidney Int. 2012 Oct;82(7):790-6.

9 Maixnerova D, Ling C, Hall S, Reily C, Brown R, Neprasova M, et al. Galactose-deficient IgA1 and the corresponding IgG autoantibodies predict IgA nephropathy progression. PLoS One. 2019 Feb;14(2):e0212254.

10 Sun Q, Zhang Z, Zhang H, Liu X. Aberrant IgA1 Glycosylation in IgA Nephropathy: A Systematic Review. PLoS One. 2016 Nov;11(11):e0166700.

11 Hiki Y, Odani H, Takahashi M, Yasuda Y, Nishimoto A, Iwase H, et al. Mass spectrometry proves under-O-glycosylation of glomerular IgA1 in IgA nephropathy. Kidney Int. 2001 Mar;59(3):1077-85.

12 Allen AC, Bailey EM, Brenchley PE, Buck KS, Barratt J, Feehally J. Mesangial IgA1 in IgA nephropathy exhibits aberrant O-glycosylation: observations in three patients. Kidney Int. 2001 Sep;60(3):969-73.

13 Yasutake J, Suzuki Y, Suzuki H, Hiura N, Yanagawa H, Makita Y, et al. Novel lectin-independent approach to detect galactose-deficient IgA1 in IgA nephropathy. Nephrol Dial Transplant. 2015 Aug;30(8):1315-21.

14 Suzuki H, Yasutake J, Makita Y, Tanbo Y, Yamasaki K, Sofue T, et al. IgA nephropathy and IgA vasculitis with nephritis have a shared feature involving galactose-deficient IgA1-oriented pathogenesis. Kidney Int. 2018 Mar; 93(3):700-5.

15 Kong X, Ma Y, Chen J, Luo Q, Yu X, Li Y, et al.; Chinese eGFR Investigation Collaboration. Evaluation of the Chronic Kidney Disease Epidemiology Collaboration equation for estimating glomerular filtration rate in the Chinese population. Nephrol Dial Transplant. 2013 Mar;28(3):641-51.

16 Oka K, Nishimura K, Kishikawa H, Ichikawa Y. IgA1 dominant subclass of latent IgA mesangial deposition in donated kidney. Int J Nephrol Renovasc Dis. 2016 Nov; 9:313-7.

17 Novak J, Julian BA, Tomana M, Mestecky J. IgA glycosylation and IgA immune complexes in the pathogenesis of IgA nephropathy. Semin Nephrol. 2008 Jan;28(1):78-87.

18 Suzuki H, Moldoveanu Z, Hall S, Brown R, Vu HL, Novak L, et al. IgA1-secreting cell lines from patients with IgA nephropathy produce aberrantly glycosylated IgA1. J Clin Invest. 2008 Feb;118(2):629-39.

19 Wada Y, Matsumoto K, Suzuki T, Saito T, Kanazawa N, Tachibana S, et al. Clinical significance of serum and mesangial galactose-deficient IgA1 in patients with IgA nephropathy. PLoS One. 2018 Nov;13(11):e0206865.

20 Floege J. IgA nephropathy: toward more specific diagnosis (and rescue of snails). Kidney Int. 2018 Mar;93(3):542-4. 
21 Xu LX, Zhao MH. Aberrantly glycosylated serum IgA1 are closely associated with pathologic phenotypes of IgA nephropathy. Kidney Int. 2005 Jul;68(1):167-72.

22 Caliskan Y, Ozluk Y, Celik D, Oztop N, Aksoy A, Ucar AS, et al. The Clinical Significance of Uric Acid and Complement Activation in the Progression of IgA Nephropathy. Kidney Blood Press Res. 2016;41(2):148-57.

23 Rizk DV, Maillard N, Julian BA, Knoppova B, Green TJ, Novak J, et al. The Emerging Role of Complement Proteins as a Target for Therapy of IgA Nephropathy. Front Immunol. 2019 Mar;10:504.

24 Floege J, Daha MR. IgA nephropathy: new insights into the role of complement. Kidney Int. 2018 Jul;94(1): 16-8.

25 Kallenberg CG, Stegeman CA, Abdulahad WH, Heeringa P. Pathogenesis of ANCA-associated vasculitis: new possibilities for intervention. Am J Kidney Dis. 2013 Dec;62(6):1176-87.

26 Ehrnthaller C, Ignatius A, Gebhard F, Huber-Lang M. New insights of an old defense system: structure, function, and clinical relevance of the complement system. Mol Med. 2011 Mar-Apr;17(3-4):317-29. 